\title{
MONOTONE DECOMPOSITIONS OF CONTINUA NOT SEPARATED BY ANY SUBCONTINUA
}

\author{
ELDON J. VOUGHT
}

\begin{abstract}
Let $M$ be a compact, metric continuum that is separated by no subcontinuum. If such a continuum has a monotone, upper semicontinuous decomposition, the elements of which have void interior and for which the quotient space is a simple closed curve, then it is said to be of type $A^{\prime}$. It is proved that a bounded plane continuum is of type $\mathrm{A}^{\prime}$ if and only if $M$ contains no indecomposable subcontinuum with nonvoid interior. In $E^{3}$ this condition is not sufficient and an example is given to illustrate this. However, it is shown that if $M$ is hereditarily decomposable then $M$ is of type $\mathrm{A}^{\prime}$. Next, a condition is given that characterizes continua of type $A^{\prime}$. Also the structure of the elements in the decomposition of a continuum of type $A^{\prime}$ is discussed and the decomposition is shown to be unique. Finally, some consequences of these results and some remarks are given.
\end{abstract}

A continuum $M$ irreducible between two points is defined by Thomas [7, p. 13] to be of type $\mathrm{A}^{\prime}$ if $M$ has a monotone, upper semicontinuous decomposition each of whose elements has void interior and whose quotient space is an arc. He proves [7, p. 15] that $M$ is of type $\mathrm{A}^{\prime}$ if and only if $M$ contains no indecomposable subcontinuum with nonvoid interior. For a continuum $M$ not separated by any subcontinuum it is natural to define $M$ to be of type $\mathrm{A}^{\prime}$ if the above definition holds except that the quotient space is required to be a simple closed curve instead of an arc. For these continua it seems reasonable too that a characterization can be obtained by using Thomas' condition. If the continuum is planar this is true and is the content of Theorem 1. But surprisingly the characterization is not valid in 3-space and Example 1 is a continuum not separated by any subcontinuum, not containing any region-containing indecomposable subcontinuum yet not of type $\mathrm{A}^{\prime}$. Interestingly enough though, if $M$ is hereditarily decomposable it is of type $\mathrm{A}^{\prime}$ and this is proved in Theorem 2 using a result of Schlais [6]. But, whereas the former condition is too weak to obtain a characterization as Example 1 shows, the latter condition is too strong as easy examples show. It is of some interest to speculate what the "right" intermediate condition is. Theorem $\mathbf{3}$ gives a characterization but the condition is not entirely satisfactory. L. E. Rogers raised the question as to whether the desired condition might be that $K(x)^{\circ}=\varnothing$ for all $x \in M$ where $K(x)$ consists of $x$ and all points $y$ of $M$

Presented to the Society, November 18, 1972 under the title Monotone decompositions of continua into simple closed curves and generalized simple closed curves; received by the editors August 9, 1972 and, in revised form, March 2, 1973.

AMS (MOS) subject classifications (1970). Primary 54F15; Secondary 54B15, 54F20.

Key words and phrases. Indecomposable continuum, continuum of type $\mathbf{A}^{\prime}$, monotone decomposition, upper semicontinuous decomposition, core decomposition, hereditarily decomposable subcontinuum, simple closed curve. 
such that $M$ is not aposyndetic at $x$ with respect to $y$. The author does not know the answer to this question. Finally some theorems are given that yield information about the structure of the elements in the decomposition of type $\mathrm{A}^{\prime}$ continua and the uniqueness of the decomposition. The author wishes to thank Leonard Rubin of the University of Oklahoma for his helpful insights and suggestions, especially in regard to Example 1.

1. Preliminary theorems. Unless stated otherwise $M$ will be a compact, metric continuum that is separated by none of its subcontinua. If $A$ is a subset of $M$ then $T(A)$ consists of $A$ together with all points $x \in M$ such that there does not exist an open set $U$ and a continuum $H$ such that $x \in U \subset H \subset M-A$. If $x \in M$ let $T^{0}(x)=x$, and $T^{n}(x)=T\left(T^{n-1}(x)\right)$ where $n$ is a positive integer. If $A$ is a subset of $M$ let $K(A)$ consist of $A$ together with all points $x \in M$ such that there does not exist an open set $U$ and continuum $H$ such that $A \subset U \subset H$ $\subset M-\{x\}$. The continuum $M$ is of type $A$ if $M$ has a monotone, upper semicontinuous decomposition whose quotient space is a simple closed curve and is of type $A^{\prime}$ if, in addition, the elements of the decomposition have void interior. If $B, C, D \subset M$ we say $B$ cuts $C$ from $D$ if every subcontinuum of $M$ intersecting both $C$ and $D$ also intersects $B$. If $P \subset M$ we say $P$ separates $M$ if $M-P$ is not connected. Finally, if $H$ is a subset of $M$ then the boundary of $H$, the interior of $H$ and the closure of $H$, relative to $M$, are denoted by $\operatorname{Fr}(H), H^{\circ}$ and $H$, respectively.

Theorem A (Thomas [7]). Let $M$ be a continuum irreducible between a pair of its points. A necessary and sufficient condition that $M$ have a monotone, upper semicontinuous decomposition, the elements of which have void interior, and for which the quotient space is an arc, is that $M$ contain no region-containing indecomposable subcontinuum.

Theorem B. A necessary and sufficient condition that $M$ be of type $A^{\prime}$ is that $M$ contain no indecomposable subcontinuum with nonvoid interior and that there exist mutually disjoint subcontinua $H, K$ such that $H^{\circ} \neq \varnothing \neq K^{\circ}$.

Proof. If $M$ is of type $\mathrm{A}^{\prime}$ then the quotient space $M^{\prime}$ is a simple closed curve. Let $x, y \in M^{\prime}$. Then $M^{\prime}-\{x, y\}=A \cup B$, a separation, where $\bar{A}=\{x\} \cup A$ $\cup\{y\}$ and $\bar{B}=\{x\} \cup B \cup\{y\}$ are arcs with endpoints $x$ and $y$. If $f$ is the quotient map of $M$ onto $M^{\prime}$ then $\overline{f^{-1}(A)}$ and $\overline{f^{-1}(B)}$ are irreducible continua from $f^{-1}(x)$ to $f^{-1}(y)$ and have monotone, upper semicontinuous decompositions whose elements have void interiors and with the arcs $\bar{A}$ and $\bar{B}$, respectively, as quotient spaces. Using Theorem A the necessity is proved.

Next suppose $M$ contains continua $H, K$ such that $H^{\circ} \neq \varnothing \neq K^{\circ}$ and $H \cap K=\varnothing$. Since $M-(H \cup K)$ is not connected (because $\overline{M-(H \cup K)}$ separates $M), M-(H \cup K)=A \cup B$, a separation of $M$. Now either $\bar{A}$ or $\bar{B}$ must contain a continuum intersecting both $H$ and $K$ (otherwise $M$ will not be connected). Let $C$ be an irreducible subcontinuum of $\bar{A}$ from $H$ to $K$. We must have $C=\bar{A}$ or else $M-(H \cup K \cup C)=(A-C) \cup B$, a separation of $M$. 
Since $H \cup A \cup K$ is a continuum, $B$ is connected. Also $\bar{B}$ must intersect both $H$ and $K$, otherwise $K$ or $H$, respectively, will separate $M$. As with $\bar{A}, \bar{B}$ is also an irreducible continuum from $H$ to $K$. By hypothesis $\bar{A}$ and $\bar{B}$ do not contain indecomposable subcontinua with nonvoid interiors; hence by Theorem $\mathrm{A}, \bar{A}$ and $\bar{B}$ have monotone upper semicontinuous decompositions the elements of which have void interiors and whose quotient spaces are arcs. Let $D_{A}$ and $D_{B}$ be elements of the decompositions of $\bar{A}$ and $\bar{B}$, respectively, such that $D_{A} \subset A$ and $D_{B} \subset B$. By the above reasoning $M-\left(D_{A} \cup D_{B}\right)=P \cup Q$, a separation of $M$, such that $\bar{P}$ and $\bar{Q}$ are both irreducible continua from $D_{A}$ to $D_{B}$. As with $\bar{A}$ and $\bar{B}$ above, $\bar{P}$ and $\bar{Q}$ have monotone, upper semicontinuous decompositions with arcs for the quotient spaces. Clearly $D_{A} \cup D_{B} \subset \bar{P} \cup \bar{Q}$. Therefore $M=\bar{P}$ $\cup \bar{Q}$ and is of type $A^{\prime}$.

The condition that there exist mutually disjoint continua with nonempty interiors is another way of saying that $M$ is not strictly nonmutually aposyndetic [3].

Theorem C. The continuum $M$ is of type $A^{\prime}$ if and only if $M$ contains no indecomposable subcontinuum with nonvoid interior and there exists $x \in M$ such that $T^{2}(x) \neq M$.

Proof. The necessity is proved as in Theorem B. Suppose $y \in M-T^{2}(x)$. By Theorem B we need to show that there exist subcontinua $H$ and $K$ such that $H^{\circ} \neq \varnothing \neq K^{\circ}$ and $H \cap K=\varnothing$. Let $H$ be a subcontinuum of $M$ such that $y \in H^{\circ} \subset H \subset M-T(x)$. For each point $z \in H$, there exists a continuum $H_{z}$ such that $z \in H_{z}^{\circ} \subset H_{z} \subset M-\{x\}$. By the compactness of $H$ and because it is connected, there exists a continuum $L$ such that $H \subset L^{\circ} \subset L \subset M-\{x\}$. The set $M-L$ is connected so if we let $K=\overline{M-L}$ it is clear that the continua $H$ and $K$ meet the required conditions.

Theorem D. The continuum $M$ is of type $A^{\prime}$ if and only if $M$ contains no indecomposable subcontinuum with nonvoid interior and there exist points $x$ and $y$ such that $T(x) \cap T(y)=\varnothing$.

Proof. Let $x, y \in M$ such that $T(x) \cap T(y)=\varnothing$. For each $z \in T(x)$ there exists a continuum $H_{z}$ such that $z \in H_{z}^{\circ} \subset H_{z} \subset M-\{y\}$. By compactness and because $T(x)$ is connected [4], there exists a continuum $H$ such that $T(x) \subset H^{\circ}$ $\subset H \subset M-\{y\}$. Let $K=\overline{M-H}$ which is a continuum since $M-H$ is connected. For each $z \in K$ there exists a continuum $K_{z}$ such that $z \in K_{z}^{\circ} \subset K_{z}$ $\subset M-\{x\}$. Again there exists a continuum $L$ such that $K \subset L^{\circ} \subset L \subset M$ $-\{x\}$. Let $N=\overline{M-L}$. Clearly $N \cap K=\varnothing$ and $N^{\circ} \neq \varnothing \neq K^{\circ}$. Therefore $M$ is of type $\mathbf{A}^{\prime}$ by Theorem $\mathbf{B}$.

2. A decomposition theorem for plane continua. In order to prove the main theorem of this section we need a corollary of a result due to Bing [1]. For completeness the proof is included. 
Lemma (Bing). If $M$ is a compact, metric continuum that is separated by no subcontinuum, then no subcontinuum of $M$ cuts between open sets.

Proof. Suppose $C$ is a subcontinuum of $M$ that cuts between the open sets $U_{1}, U_{2}$. Let $V_{1}, V_{2}$ be nonempty open sets such that $\bar{V}_{1} \subset U_{1}, \bar{V}_{2} \subset U_{2}$. Let $C^{\prime}$ be the component of $M-\left(V_{1} \cup V_{2}\right)$ that contains $C$. Each component of $\boldsymbol{M}$ $-\left(V_{1} \cup V_{2}\right)$ must intersect $\bar{V}_{1}-V_{1}$ or $\bar{V}_{2}-V_{2}$ but none, except $C^{\prime}$, intersects both since such a continuum would intersect $U_{1}$ and $U_{2}$ but not $C$. For each point $a \in M-\left(V_{1} \cup V_{2}\right)$ let $Q_{a}$ be the component of $M-\left(V_{1} \cup V_{2}\right)$ that contains $a$, let

$$
Q_{1}=\cup\left\{Q_{a} \mid a \in M-\left(V_{1} \cup V_{2}\right) ; Q_{a} \neq C^{\prime} ; Q_{a} \cap\left(\nabla_{1}-V_{1}\right) \neq \varnothing\right\}
$$

and

$$
Q_{2}=\cup\left\{Q_{a} \mid a \in M-\left(V_{1} \cup V_{2}\right) ; Q_{a} \neq C^{\prime} ; Q_{a} \cap\left(\bar{V}_{2}-V_{2}\right) \neq \varnothing\right\} .
$$

As noted $Q_{1} \cap Q_{2}=\varnothing$; furthermore $\bar{Q}_{1} \cap Q_{2}=\varnothing$. For suppose $p \in \bar{Q}_{1} \cap Q_{2}$. Then there exists a sequence of components $Q_{a_{1}}, Q_{a_{2}}, \ldots$, such that $Q_{a_{i}} \subset Q_{1}$ for each $i$, and $p \in \lim \inf Q_{a_{i}}$. Then $\lim \sup Q_{a_{i}}$ is a continuum $K$ that lies in the component $Q_{p}$ of $M-\left(V_{1} \cup V_{2}\right)\left(Q_{p} \neq C^{\prime}\right.$ since $\left.p \notin C^{\prime}\right)$. Now $p \in Q_{2}$ so $Q_{p} \subset Q_{2}$; but $K \cap\left(\bar{V}_{1}-V_{1}\right) \neq \varnothing$, so $Q_{p} \subset Q_{1}$. This is impossible and therefore $\bar{Q}_{1} \cap Q_{2}=\varnothing$; similarly $Q_{1} \cap \bar{Q}_{2}=\varnothing$. Let $A=V_{1} \cup Q_{1}$ and $B=V_{2} \cup Q_{2}$. We have $M-C^{\prime}=A \cup B$ and $\bar{A} \cap B=\varnothing=A \cap \bar{B}$. Thus $M-C^{\prime}$ is not connected which is a contradiction since $C^{\prime}$ is a subcontinuum of $M$.

We now prove the simple closed curve decomposition theorem for plane continua.

Theorem 1. If $M$ is a bounded plane continuum that is separated by none of its subcontinua and contains no region-containing indecomposable subcontinua, then $\boldsymbol{M}$ is of type $A^{\prime}$ and conversely.

Proof. The converse is proved in general in Thereom B. According to Theorem B it is sufficient to prove that there exist two subcontinua $H, K$ with nonempty interiors such that $H \cap K=\varnothing$. Since $M$ contains no indecomposable subcontinuum with nonempty interior and no subcontinuum separates $M$, there exist subcontinua $M_{1}, M_{2}, M_{3}, M_{4}, M_{5}$ whose interiors relative to $M$ are nonempty and pairwise disjoint and such that $M=M_{1} \cup M_{2} \cup M_{3} \cup M_{4} \cup M_{5}$. Suppose that $M_{i} \cap M_{j} \neq \varnothing ; 1 \leq i, j \leq 5$. There exist pairwise disjoint simple closed disks $J_{1}$, $J_{2}, J_{3}, J_{4}, J_{5}$ such that $J_{i}^{\circ} \cap M_{i} \neq \varnothing, i=1,2,3,4,5 ; J_{i} \cap M_{j}=\varnothing, i \neq j\left(J_{i}^{\circ}\right.$ is the interior of $J_{i}$ in the plane). The continuum $M_{3} \cup M_{4} \cup M_{5}$ does not separate $M$ and hence does not cut $M$ between the open sets $J_{1}^{\circ} \cap M_{1}$ and $J_{2}^{\circ} \cap M_{2}$ of $M$. Hence there exists a subcontinuum $B_{12}$ of $M-\left(M_{3} \cup M_{4} \cup M_{5}\right)$ intersecting these two sets. Let $C_{12}$ be an irreducible subcontinuum of $B_{12}$ from $J_{1}$ to $J_{2}$. Clearly $C_{12} \cap J_{1}^{\circ}=\varnothing=C_{12} \cap J_{2}^{\circ}$. The continuum $M_{2} \cup M_{4} \cup M_{5} \cup C_{12}$ does not cut $M$ between the open sets $J_{1}^{\circ} \cap M_{1}$ and $J_{3}^{\circ} \cap M_{3}$. There exists a subcontinuum $B_{13}$ of $M-\left(M_{2} \cup M_{4} \cup M_{5} \cup C_{12}\right)$ intersecting these sets and an irreduc- 
ible subcontinuum $C_{13}$ of $B_{13}$ from $J_{1}$ to $J_{3}$. As before $C_{13} \cap J_{1}^{\circ}=\varnothing=C_{13}$ $\cap J_{3}^{\circ}$. In a similar manner there exists a subcontinuum $C_{14}$ of $M-\left(M_{2} \cup M_{3}\right.$ $\left.\cup M_{5} \cup C_{12} \cup C_{13}\right)$ irreducible between $J_{1}$ and $J_{4}$ with $C_{14} \cap J_{1}^{\circ}=\varnothing=C_{14}$ $\cap J_{4}^{\circ}$. Continuing in this way we obtain for all 10 combinations of subscripts $i, j, 1 \leq i<j \leq 5$, pairwise disjoint continua $C_{i j}$ each of which is irreducible between $J_{i}$ and $J_{j}$ and intersects only these two of the five simple closed disks. For each $C_{i j}$ an $\operatorname{arc} A_{i j}$ can be constructed so that the ten $\operatorname{arcs} A_{i j}, 1 \leq i<j \leq 5$, are pairwise disjoint, $A_{i j}$ is irreducible between $J_{i}$ and $J_{j}$ and intersects only these two disks. Now if we decompose the plane by considering each $J_{i}$ to be a "point", the plane continuum $\bigcup_{i=1}^{5} J_{i} \cup \cup_{1 \leq i<j \leq 5} A_{i j}$ is topologically equivalent to the continuum of $[5$, p. 230 , second figure] which is not embeddable in the plane. So the assumption that $M_{i} \cap M_{j} \neq \varnothing, 1 \leq i, j \leq 5$, has led to a contradiction and therefore there exist two integers $i, j, 1 \leq i, j \leq 5$, such that $M_{i} \cap M_{j}=\varnothing$. The continua $M_{i}$ and $M_{j}$ have nonvoid interiors relative to $M$ and the proof is complete.

3. A continuum not of type $\mathbf{A}^{\prime}$. The following example is a continuum $M$ that is not separated by any subcontinuum (in fact, not by any finite number of subcontinua), does not contain any indecomposable subcontinuum with nonvoid interior, but is not of type $\mathbf{A}^{\prime}$.

Example 1. Let $K$ be an indecomposable plane continuum lying in the unit square and intersecting $y=0$ and $y=1$ each in a single point. Let $C$ be the Cantor middle third discontinuum and form the cartesian product $C \times K$. Each point in $C \times K$ is of the form $(x,(y, z))$ where $x \in C$ and $(y, z) \in K$ (for simplicity of notation we will write $(x, y, z)$ for $(x,(y, z)))$. Pick the point $\left(y_{1}, 1\right) \in K$ and "identify" all the points of $C \times\left\{\left(y_{1}, 1\right)\right\}$. Pick the point $\left(y_{0}, 0\right) \in K$ and identify the two points $\left(1 / 3, y_{0}, 0\right)$ and $\left(2 / 3, y_{0}, 0\right)$. Consider the six points $1 / 9,2 / 9,1 / 3,2 / 3,7 / 9,8 / 9 \in C$ and the 15 combinations taking two at a time. Pick 15 points $\left(y_{1 / 2,1}, 1 / 2\right), \ldots,\left(y_{1 / 2,15}, 1 / 2\right)$ each from a different composant of $K$ and also from different composants than those that contain $\left(y_{1}, 1\right)$ and $\left(y_{0}, 0\right)$. Make the following 15 identifications:

$$
\begin{array}{ccc}
\left(1 / 9, y_{1 / 2,1}, 1 / 2\right) & \text { and } & \left(2 / 9, y_{1 / 2,1}, 1 / 2\right), \\
\left(1 / 9, y_{1 / 2,2}, 1 / 2\right) & \text { and } & \left(1 / 3, y_{1 / 2,2}, 1 / 2\right), \\
\left(1 / 9, y_{1 / 2,3}, 1 / 2\right) & \text { and } & \left(2 / 3, y_{1 / 2,3}, 1 / 2\right), \\
& \vdots & \\
\left(7 / 9, y_{1 / 2,15}, 1 / 2\right) & \text { and } & \left(8 / 9, y_{1 / 2,15}, 1 / 2\right) .
\end{array}
$$

Next consider the 14 points $1 / 27,2 / 27,1 / 9,2 / 9,7 / 27,8 / 27,1 / 3,2 / 3,19 / 27$, $20 / 27,7 / 9,8 / 9,25 / 27,26 / 27$ and the 91 combinations taking two at a time. Pick 91 points $\left(y_{2 / 3,1} 2 / 3\right), \ldots,\left(y_{2 / 3,91}, 2 / 3\right)$ each from a different composant of $K$ and also from different composants than those chosen earlier. Make 91 identifications: 


$$
\begin{gathered}
\left(1 / 27, y_{2 / 3,1}, 2 / 3\right) \text { and }\left(2 / 27, y_{2 / 3,1}, 2 / 3\right), \\
\left(1 / 27, y_{2 / 3,2}, 2 / 3\right) \text { and }\left(1 / 9, y_{2 / 3,2}, 2 / 3\right), \\
\left(1 / 27, y_{2 / 3,3}, 2 / 3\right) \text { and }\left(2 / 9, y_{2 / 3,3}, 2 / 3\right), \\
\vdots \\
\left(25 / 27, y_{2 / 3,2 / 3,91}, 2 / 3\right) \text { and }\left(26 / 27, y_{2 / 3,91}, 2 / 3\right) .
\end{gathered}
$$

Continue inductively this construction of identification of pairs of points. Let $M$ be the quotient space of the decomposition of $C \times K$ whose elements consist of all these identifications (this includes $C \times\left\{\left(y_{1}, 1\right)\right\}$ as one of the elements) together with the remaining individual points of $C \times K$. This decomposition is clearly upper semicontinuous and $M$ is a compact, metric continuum. Notice that any infinite sequence of $M$ whose elements are identified pairs must converge to the point $C \times\left\{\left(y_{1}, 1\right)\right\}$ which, for brevity, will be denoted by $p$ hereafter. Let $U=\{(x, y, z) \in C \times K \mid z \neq 1$ and $x$ is not an endpoint in $C\}$ and $Q=M$ - $(U \cup\{p\})$. ( $Q$ contains all the identified pairs and individual points $(x, y, z)$ of $C \times K$ where $x$ is an endpoint in $C$.) So $M=\{p\} \cup U \cup Q$.

(1) Let us show that $p$ cuts $U$ from $Q$ in $M$ and cuts any two points of $U$ with unequal $x$-coordinates. Let $M^{\prime}$ be the quotient space of $C \times K$ formed by identifying all the points of $C \times\left\{\left(y_{1}, 1\right)\right\}$ and regarding all other points of $C \times K$ as individual elements of the decomposition. Clearly the point $p=C \times\left\{\left(y_{1}, 1\right)\right\}$ in $M^{\prime}$ cuts any two points of $M^{\prime}$ with unequal $x$-coordinates. But this implies that $p$ cuts $U$ from $Q$ in $M$ and also cuts two points of $U$ with unequal $x$-coordinates.

(2) Let $\alpha$ be a nonendpoint of the Cantor set and let $U_{\alpha}=(\{\alpha\} \times K)-\{p\}$ in $M$. If $H$ is any subcontinuum of $M$ that contains $p$ then $H \cap\left(U_{\alpha} \cup\{p\}\right)$ is a continuum. For suppose the closed set $H \cap\left(U_{\alpha} \cup\{p\}\right)=A \cup B$, a separation, with $p \in A$. Let $V, W$ be open sets in $M$ such that $A \subset V, B \subset W$ and $V \cap W=\varnothing$. Choose a point $b \in B$. Since $H$ is a continuum then $\operatorname{Fr}(W) \cap H$ $\neq \varnothing$ and so there exists a subcontinuum $N$ of $H \cap \bar{W}$ such that $b \in N$ and $N \cap \operatorname{Fr}(W) \neq \varnothing$. Now $N \subset H$ but $N \nsubseteq U_{\alpha}$ for otherwise $N \subset A \cup B$ which implies that $\operatorname{Fr}(W) \cap(A \cup B) \neq \varnothing$. So $N \subset M-\{p\}, N \cap U_{\alpha} \neq \varnothing$ and $N \nsubseteq U_{\alpha}$. From (1) this is impossible.

(3) We will now prove that $T^{2}(x)=M$ for all $x \in M$. Clearly $T(p)=M$ from (1) and so $T^{2}(p)=M$. Let $x \in M-\{p\}$ and let us show that $p \in T(x)$. Then since $T(p) \subset T^{2}(x)$ and $T(p)=M$ it will follow that $T^{2}(x)=M$. Suppose $p \notin T(x)$. Then there exists an open set $W$ and continuum $H$ such that $p \in W \subset H \subset M-\{x\}$. Let $\alpha$ be a nonendpoint of the Cantor set $C$ such that $U_{\alpha}$ intersects $M-H$. From (2), $H \cap\left(U_{\alpha} \cup\{p\}\right)$ is a continuum. Also $W$ $\cap\left(U_{\alpha} \cup\{p\}\right)$ is an open set in $U_{\alpha} \cup\{p\}$ containing $p$. So we have the sequence of inclusions $p \in W \cap\left(U_{\alpha} \cup\{p\}\right) \subset H \cap\left(U_{\alpha} \cup\{p\}\right) \subset U_{\alpha} \cup\{p\}$. Therefore $H \cap\left(U_{\alpha} \cup\{p\}\right)$ is a proper subcontinuum with nonvoid interior of the indecomposable continuum $U_{\alpha} \cup\{p\}$. This contradiction shows that $p \in T(x)$. 
(4) No indecomposable subcontinuum with nonvoid interior is contained in $M$. To see this let $H$ be such a subcontinuum of $M$. It follows from (1) that $p \in H$. For each nonendpoint $\alpha \in C$ such that $U_{\alpha} \cap H \neq \varnothing$ by (2) we have that $H \cap\left(U_{\alpha} \cup\{p\}\right)$ is a continuum. Hence a single composant of $H$ contains all points of $U \cap H$. If $\alpha$ is an endpoint of $C$ and $Q_{\alpha}=(\{\alpha\} \times K)-\{p\}$ then in $M$ the set $H \cap\left(Q_{\alpha} \cup\{p\}\right)$ has at most a countable number of components. This is true because any such component must contain $p$ or a point of $Q_{\alpha}$ which is one of the identified pairs and the set of these identified pairs in $Q_{\alpha}$ is countable. The set of endpoints of $C$ is countable and so $H \cap Q$ is the union of a countable number of continua. Therefore $H$ has a countable number of composants contradicting the assumption that $H$ is indecomposable.

(5) Next it will be established that no subcontinuum of $M$ separates $M$. Let $H$ be a proper subcontinuum of $M$. If $H$ does not contain $p$ then it is clear from (1) that $H$ does not separate $M$. Suppose $p \in H$. If $Q \subset H$ then $M=\bar{Q} \subset H$. So let $Q^{\prime}=\left\{Q_{\alpha} \mid Q_{\alpha} \nsubseteq H\right\}$ and assume $Q^{\prime}$ is not empty. If $Q_{\alpha} \in Q^{\prime}$ and $H$ contains a point $x$ of $Q_{\alpha}$ that is an identified pair in the decomposition of $C \times K$ then $H$ must contain the entire "dual" continuum $Q_{\beta} \cup\{p\}$, i.e., the entire continuum $Q_{\beta} \cup\{p\}$ that contains the other point of the identified pair. For if not then neither $H \cap\left(Q_{\alpha} \cup\{p\}\right)$ nor $H \cap\left(Q_{\beta} \cup\{p\}\right)$ can have a subcontinuum containing both $x$ and $p$. Then each set can be separated into disjoint closed sets, one containing $p$ and the other $x$. But by using (1) and the argument in (2), a contradiction is obtained. Clearly if $x$ is an identified pair in $Q_{\alpha}$ and $x \notin H$ then the "dual" continuum $Q_{\beta} \cup\{p\} \nsubseteq H$. Choose any $Q_{\alpha} \in Q^{\prime}$. Now all the points of $Q_{\alpha}$ such that $x$ is an identified pair in $C \times K$ cannot belong to $H$ or else $H$ would contain all the "dual" continua of these points. But this would include all points of $Q$ and thus $H=M$ since $\bar{Q}=M$, a contradiction to the fact that $H$ is a proper subcontinuum. Let $X$ be all points $x$ of $Q_{\alpha}$ that are identified pairs in the decomposition but are not contained in $H$. For any element $Q_{\alpha^{\prime}} \in Q^{\prime}$, it must be that $Q_{\alpha^{\prime}}-H$ is connected since $H$ cannot intersect any composant of $Q_{\alpha^{\prime}} \cup\{p\}$ that does not contain $p$ or one of the countable number of identified pairs in $Q_{\alpha^{\prime}}$. So $Q_{\alpha}-H$ is connected and so is $Q_{\beta}-H$ where $Q_{\beta} \cup\{p\}$ is any one of the dual continua of $Q_{\alpha}$ for some point $x \in X$. Now $Q-H=\left(Q_{\alpha}-H\right)$ $\cup \cup_{x \in X}\left(Q_{\beta}-H\right)$ and this set is connected since $\left(Q_{\alpha}-H\right) \cup\left(Q_{\beta}-H\right)$ is connected for each $x \in X$. But $Q-H$ is certainly dense in $M-H$ and so $M-H$ is connected. This concludes the proof that no subcontinuum separates $M$ and, in fact, it follows easily that no finite number of subcontinua separate $M$.

It has now been established that $M$ is a continuum that is separated by no subcontinuum, contains no indecomposable subcontinuum with nonvoid interior, and $T^{2}(x)=M$ for all $x \in M$. By Theorem $\mathrm{C}, M$ is not of type $\mathrm{A}^{\prime}$.

4. Conditions for type $\mathbf{A}^{\prime}$ continua. The first theorem in this section depends on a theorem due to Schlais [6]. Schlais states his theorem for a point $x$ but the proof goes through without any difficulty if $x$ is replaced by a continuum $H$ with void 
interior and will be stated here in this more general setting. The theorem is valid for any compact, metric continuum.

Theorem (Schlais). Let $M$ be a compact, metric continuum. If $M$ is hereditarily dcomposable then for every subcontinuum $H$ with void interior, $K(H)^{\circ}=\varnothing$.

Theorem 2. Let $M$ be a compact, metric continuum not separated by any subcontinuum. If $M$ is hereditarily decomposable then $M$ is of type $A^{\prime}$.

Proof. Because no continuum separates it follows immediately that for any closed set $F$ we have $K(F)=T(F)$. Let $x \in M$. By Schlais' theorem we have $T(x)^{\circ}=K(x)^{\circ}=\varnothing$ and consequently $T^{2}(x)^{\circ}=T(T(x))^{\circ}=K(T(x))^{\circ}=\varnothing$ since $T(x)$ is a continuum with void interior. Since $T^{2}(x)$ has empty interior, $T^{2}(x) \neq M$, and hence by Theorem C, $M$ is of type $\mathrm{A}^{\prime}$.

That the condition in Theorem 2 is not necessary for $M$ to be of type $\mathrm{A}^{\prime}$ is shown by the following example.

Example 2. Let $D$ be the indecomposable "semicircle" continuum on p. 143 of Kuratowski's book [5] which has only one endpoint. Let $R$ be a ray. The ray $R$ can be "wrapped" in such a way that $D \cap R=\{x\}$ where $x$ is the common endpoint of both sets, and $D$ is the limiting set of $R$. Let $M=D \cup R$. Clearly no subcontinuum separates $M$ and $M$ is of type $\mathrm{A}^{\prime}$ where the only nondegenerate element of the decomposition is the indecomposable continuum $D$.

An interesting consequence of Theorem 2 is that a hereditarily decomposable continuum $M$ is separated by some two of its subcontinua.

Corollary. Let $M$ be a compact, metric, hereditarily decomposable continuum. There exist subcontinua $C_{1}$ and $C_{2}$ such that $M-\left(C_{1} \cup C_{2}\right)$ is not connected.

Proof. Suppose not. If no pair of subcontinua separates $M$ then certainly no subcontinuum separates $M$. By Theorem 2, $M$ is of type $\mathrm{A}^{\prime}$. Let $D_{a}$ and $D_{b}$ be any two distinct elements of the decomposition. Clearly $D_{a} \cup D_{b}$ separates $M$, a contradiction.

As the previous theorems and examples show, hereditarily decomposable is too much and the exclusion of region-containing indecomposable continua is too little to characterize type $\mathrm{A}^{\prime}$ continua. Theorem 3 uses an intermediate condition to provide a characterization although probably not the "best" one.

Theorem 3. Let $M$ be a compact, metric continuum not separated by any subcontinuum. If for every subcontinuum $H$ with void interior it follows that $T(H)^{\circ}=\varnothing$ then $M$ is of type $A^{\prime}$ and conversely.

Proof. Suppose $M$ is of type $\mathrm{A}^{\prime}$ and let $H$ be a subcontinuum of $M$ with empty interior. If $H \cap f^{-1}(x) \neq \varnothing \neq H \cap f^{-1}(y), x \neq y$, where $f$ is the map from $M$ onto the quotient space $M^{\prime}$, then $M-\left(f^{-1}(x) \cup f^{-1}(y)\right)=A \cup B$, a separation of $M$, where $\bar{A}$ and $\bar{B}$ are irreducible subcontinua of $M$ from $f^{-1}(x)$ to $f^{-1}(y)$. Let 
$H^{\prime}$ be an irreducible subcontinuum of $H$ from $f^{-1}(x)$ to $f^{-1}(y)$. Either $H^{\prime} \subset \bar{A}$ or $H^{\prime} \subset \bar{B}$ so let us assume that $H^{\prime} \subset \bar{A}$. It follows that $H^{\prime}=\bar{A}$ since $\bar{A}$ is irreducible from $f^{-1}(x)$ to $f^{-1}(y)$. So $H^{\prime}$ contains the open set $A$. But $H$ contains no such open set; hence there exists $x \in M^{\prime}$ such that $H \subset f^{-1}(x)$. Suppose there exists $y \in M^{\prime}$ such that $f^{-1}(y) \cap T(H) \neq \varnothing, x \neq y$. Since $M^{\prime}$ is a simple closed curve there exist $u, v \in M^{\prime}$ such that $\{u\} \cup\{v\}$ separates $x$ from $y$ in $M^{\prime}$. Then $f^{-1}(u) \cup f^{-1}(v)$ separates $M$ into sets $A$ and $B$ with $f^{-1}(x) \subset A$ and $f^{-1}(y) \subset B$. But $f^{-1}(u) \cup B \cup f^{-1}(v)$ is a continuum $L$ that contains a point of $T(H)$ in its interior. However $H \subset f^{-1}(x) \subset M-L$ which is a contradiction. Hence $T(H) \subset f^{-1}(x)$. Since $f^{-1}(x)^{\circ}=\varnothing$ then $T(H)^{\circ}=\varnothing$.

Next suppose $T(H)^{\circ}=\varnothing$ for every continuum $H$ where $H^{\circ}=\varnothing$. If $x \in M$ then $T(x)^{\circ}=\varnothing$ and since $T(x)$ is a continuum, $T^{2}(x)^{\circ}=T(T(x))^{\circ}=\varnothing$. Therefore $T^{2}(x) \neq M$ and we will complete the proof that $M$ is of type $\mathrm{A}^{\prime}$ by showing that $M$ contains no indecomposable subcontinuum with nonvoid interior and applying Theorem C. Let $K$ be an indecomposable continuum with nonempty interior lying in $M$. Let $x, y \in K^{\circ}$. Suppose there is a continuum $P$ and an open set $U$ such that $y \in U \subset P \subset M-\{x\}$. Since $K$ is indecomposable $P \nsubseteq K$. Therefore $P$ intersects the connected set $M-K$ and so $M-R \cup P$ is a continuum $Q$ such that $y \in Q^{\circ}, x \notin Q$. We have $x \in M-Q \subset \overline{M-Q}$ $\subset K-\{y\}$. But $M-Q$ is connected and so $\overline{M-Q}$ is a proper subcontinuum of $K$ with nonvoid interior which is impossible. This means that $y \in T(x)$ and since $y$ was chosen arbitrarily it follows that $K^{\circ} \subset T(x)$ contradicting the hypothesis that $T(x)^{\circ}=\varnothing$.

Question. In Theorem 3 can "subcontinuum $H$ " be replaced by "point $x$ "?

5. Element structure and uniqueness of the decomposition of type $A^{\prime}$ continua. For a compact, Hausdorff continuum $M$, FitzGerald and Swingle [2, p. 39] have proved that if $\left\{T^{n}(x) \mid x \in M\right\}$ is a decomposition of $M$ and $T^{n}(x)=T^{n+1}(x)$ for all $x \in M$, then $\left\{T^{n}(x) \mid x \in M\right\}$ is the core decomposition of $M$ with respect to being monotone, upper semicontinuous and having a semilocally connected quotient space $M^{\prime}$. By the core decomposition is meant a decomposition of $M$ that has the aforementioned properties and refines every other decomposition with these properties. If a continuum $M$ in which no subcontinuum separates has a monotone, upper semicontinuous decomposition with a semilocally connected quotient space $M^{\prime}$, then clearly no subcontinuum separates $M^{\prime}$, and in such continua semilocal connectedness is equivalent to local connectedness [1, Theorem 2]. If $M$ is also metric then $M^{\prime}$ is a locally connected, metric continuum that is not separated by any subcontinuum. Hence $M^{\prime}$ is either degenerate or a simple closed curve. We can use these results next.

Theorem 4. Let $M$ be a compact, metric continuum of type $A^{\prime}$. Then $\left\{T^{2}(x) \mid x\right.$ $\in M\}$ is a monotone, upper semicontinuous decomposition of $M$, the elements of which have void interiors and for which the quotient space is a simple closed curve. 
Furthermore the decomposition is unique with respect to these properties, i.e., a type $A^{\prime}$ continuum has only one such decomposition.

Proof. Since $M$ is of type $\mathrm{A}^{\prime}$ it contains no indecomposable subcontinuum with nonvoid interior by Theorem B. First let us show that $T^{2}(x)=T^{3}(x)$ for all $x \in M$. Suppose $y \in T^{3}(x)-T^{2}(x)$. Then there is a continuum $H$ such that $y \in H^{\circ} \subset H \subset M-T(x)$. By the compactness argument used earlier there exists a continuum $L$ such that $H \subset L^{\circ} \subset L \subset M-\{x\}$. Let $N=\overline{M-L}$ which is a continuum. We have $H \cap N=\varnothing$ so $M-(H \cup N)=P \cup Q$, a separation of $M$, such that $\bar{P}$ and $\bar{Q}$ are irreducible subcontinua from $H$ to $N$. The interiors of these continua are nonempty so they are decomposable. Let $\bar{P}=P_{1} \cup P_{2}$ where $P_{1}$ and $P_{2}$ are continua for which $P_{1} \cap H \neq \varnothing \neq P_{2} \cap N$ and $P_{1} \cap N=\varnothing=P_{2} \cap H$. Let $Q_{1}$ and $Q_{2}$ be corresponding continua for $\bar{Q}$. It follows that $x \in N^{\circ}, T(x) \subset N, T^{2}(x) \subset P_{2} \cup N \cup Q_{2}, T^{3}(x) \subset\left(P_{1} \cup P_{2}\right)$ $\cup N \cup\left(Q_{1} \cup Q_{2}\right)=\bar{P} \cup N \cup \bar{Q}$. But $y \in T^{3}(x)$ yet $y \notin \bar{P} \cup N \cup \bar{Q}$, a contradiction.

Since no subcontinuum separates $M$, it follows immediately that $x \in T^{2}(y)$ if and only if $y \in T^{2}(x)$. Let $z \in T^{2}(x)$. Then $T(z) \subset T\left(T^{2}(x)\right)=T^{3}(x)$ $=T^{2}(x)$ and $T^{2}(z)=T(T(z)) \subset T\left(T^{2}(x)\right)=T^{2}(x)$. Likewise $x \in T^{2}(z)$ and we have $T^{2}(x) \subset T^{2}(z)$. Hence $T^{2}(x)=T^{2}(z)$ and $\left\{T^{2}(x) \mid x \in M\right\}$ is a decomposition of $M$. By the result of FitzGerald and Swingle and the observations immediately following, this monotone decomposition is upper semicontinuous, has a simple closed curve for a quotient space and refines every other such decomposition. Because $M$ is of type $A^{\prime}$ it follows then that $T^{2}(x)$ has void interior for all $x \in M$.

To see that $\left\{T^{2}(x) \mid x \in M\right\}$ is the only such decomposition suppose that $G$ is a different monotone, upper semicontinuous decomposition of $M$, the elements of which have void interiors, and with a simple closed curve for the quotient space. Since $\left\{T^{2}(x) \mid x \in M\right\}$ refines $G$ there is $g \in G$ and $T^{2}(x)$ for some $x \in M$ such that $T^{2}(x) \subsetneq g$. Let $y \in g-T^{2}(x)$. By the argument above there exist continua $H, N$ such that $x \in H^{\circ}, y \in N^{\circ}$ and $H \cap N=\varnothing$. Also $M-(H \cup N)=P \cup Q$, a separation of $M$. But $g \cap H \neq \varnothing \neq g \cap N$ so there exists an irreducible subcontinuum $g^{\prime}$ of $g$ from $H$ to $N$. Certainly $g^{\prime}$ contains $P$ or $Q$. But these are open sets and $g$ contains no such set so the proof is complete.

An interesting consequence of this theory is a generalization of sorts of a theorem due to Bing [1, Theorem 10].

Theorem (Bing). Let $M$ be a compact, metric continuum that is separated by no subcontinuum. If $M$ is not cut by $x$ for any $x \in M$, then $M$ is a simple closed curve.

Theorem 5. Let $M$ be a compact, metric continuum that is separated by no subcontinuum. If $M$ is not cut by $T(x)$ for any $x \in M$, then $M$ is either 
indecomposable or admits a monotone, upper semicontinuous decomposition whose quotient space is a simple closed curve (type A continuum).

Proof. First let us show that $T(x)=T^{2}(x)$ for every $x \in M$. Suppose $q \in T^{2}(x)-T(x)$. Since $T(x)$ does not cut $M$ it is easy to show by the Baire theorem that there exists a continuum $H$ such that $q \in H, H^{\circ} \neq \varnothing$ and $H \cap T(x)=\varnothing$ (we canot assume though that $q \in H^{\circ}$ ). By compactness once again there exists a continuum $L$ such that $H \subset L^{\circ} \subset L \subset M-\{x\}$. Let $N=\overline{M-L}$. We have a separation of $M, M-(H \cup N)=P \cup Q$ such that $\bar{P}$ and $\bar{Q}$ are irreducible continua from $H$ to $N$. We have $T(x) \subset N$ and $T^{2}(x)$ $\cap H^{\circ}=\varnothing$. Because $q \in T^{2}(x), q \notin H^{\circ}$ and so $q \in H \cap \bar{P}$ or $q \in H \cap \bar{Q}$. Now $\bar{P}$ and $\bar{Q}$ are disjoint so assume $q \in H \cap \bar{P}$. Since $q$ is in the interior of the continuum $H \cup \bar{P}$ it must be that $T(x) \cap \bar{P} \neq \varnothing$. It follows from the Baire theorem and the assumption that $T(x)$ does not cut between points of $M$ that there is a continuum $R$ containing $q$ and an open set $U$ of $\bar{P}$ with $U \subset R$ $\subset M-T(x)$. The closure of $S=\bar{P}-R$ is a proper subcontinuum of $\bar{P}$ containing $q$ (since $\left.q \in T^{2}(x)\right)$ and $\bar{S} \cap T(x) \neq \varnothing$. This contradicts the fact that $\bar{P}$ is irreducible from $H$ to $N$.

As in the previous argument it follows that $\{T(x) \mid x \in M\}$ is a decomposition of $M$. Using the result of FitzGerald and Swingle again, this decomposition is the core decomposition with respect to the properties of being monotone, upper semicontinuous and having a locally connected quotient space $M^{\prime}$. If $M$ is indecomposable then obviously $M^{\prime}$ is degenerate. If $M$ is decomposable where $M=M_{1} \cup M_{2}$, let $x \in M-M_{2}$ and $y \in M-M_{1}$. Then $T(x) \subset M_{1}$ and $T(y)$ $\subset M_{2}$ so $M^{\prime}$ has more than one element and must be a simple closed curve.

Although $M$ is of type A (unless it is indecomposable) it is not necessarily of type $\mathrm{A}^{\prime}$ and the next example shows this.

Example 3. Let $D$ be the indecomposable continuum of Example 2 and let $R$ be an arc $[a, b]$ such that $D \cap R=\{a, b\}$ where $a$ and $b$ lie in different composants of $D$. If $M=D \cup R$ it is clear that $M$ is of type A but not type $\mathrm{A}^{\prime}$.

The crucial notion used in Theorem 5 is a special case of our last theorem.

Theorem 6. Let $x$ be a point of a compact, metric continuum that is separated by no subcontinuum. Then $T^{n}(x)$ does not cut $M$ if and only if $T^{n}(x)=T^{n+1}(x)$ where $n$ is a positive integer.

Proof. We will prove only the sufficiency since the necessity was proved in Theorem 5 for $n=1$ and the argument is the same in general. Suppose $T^{n}(x)=T^{n+1}(x)$ and take $y, z \in M-T^{n}(x)$. Since $y, z \notin T^{n+1}(x)$ there are continua $H_{y}$ and $H_{z}$ such that $y \in H_{y}^{\circ} \subset H_{y}, z \in H_{z}^{\circ} \subset H_{z}$ and $H_{y} \cap T^{n}(x)$ $=\varnothing=H_{z} \cap T^{n}(x)$. If $H_{y} \cap H_{z} \neq \varnothing$ then $T^{n}(x)$ does not cut $y$ from $z$. If $H_{y} \cap H_{z}=\varnothing$ then $M-\left(H_{y} \cup H_{z}\right)=C_{1} \cup C_{2}$, a separation, where $\bar{C}_{1}$ and $\bar{C}_{2}$ are irreducible continua from $H_{y}$ to $H_{2}$. Inasmuch as $T^{n}(x)$ is connected we can 
assume that $T^{n}(x) \subset C_{1}$. Then $y, z \in H_{y} \cup C_{2} \cup H_{z} \subset M-T^{n}(x)$ and $T^{n}(x)$ does not cut $y$ from $z$. Since $y, z$ are arbitrary points of $M-T^{n}(x)$ the conclusion follows.

If we allow the case $n=0$ in Theorem 6 , the sufficiency holds but the necessity fails. The following example shows this.

Example 4. Let $S_{1}=\{(x, y) \mid 0<x \leq 2 / \pi, y=-1+\sin (1 / x)\}, S_{2}=\{(x, y) \mid$ $x=0,-2 \leq y \leq 0\}, S_{3}=\left\{(x, y) \mid(x-1 / \pi)^{2}+y^{2}=1 / \pi^{2}, y \geq 0\right\}$. Let $S=S_{1}$ $\cup S_{2} \cup S_{3}$ and let $x=(0,-2)$. Then $T^{0}(x)=x$ and $x$ does not cut $S$. However $S_{2}=T^{1}(x) \neq T^{0}(x)$.

But if $T^{0}(x)=x$ does not cut in $M$ for every $x \in M$, then $M$ is a simple closed curve by the theorem of Bing above. It follows that $T^{1}(x)=T^{0}(x)$ for every $x \in M$.

Remarks. The metric condition is not necessary in Theorems 3 and 4. However, for the theorems to apply to a compact, Hausdorff continuum, wherever a simple closed curve occurs a generalized simple closed curve must replace it. A compact, Hausdorff continuum is a generalized simple closed curve if it is separated by the omission of any two of its points. The decompositions involved in these theorems will now have generalized simple closed curves for quotient spaces.

\section{REFERENCES}

1. R. H. Bing, Some characterizations of arcs and simple closed curves, Amer. J. Math. 70 (1948), 497-506. MR 10, 55.

2. R. W. FitzGerald and P. M. Swingle, Core decompositions of continua, Fund. Math. 61 (1967), 33-50. MR 36 \#7110.

3. C. L. Hagopian, Mutual aposyndesis, Proc. Amer. Math. Soc. 23 (1969), 615-622. MR 40 \#876.

4. F. B. Jones, Concerning non-aposyndetic continua, Amer. J. Math. 70 (1948), 403-413. MR 9, 606.

5. K. Kuratowski, Topologie. Vol. 2, 3rd ed., Monografie Mat., Tom 21, PWN, Warsaw, 1961; English transl., Academic Press, New York; PWN, Warsaw, 1968. MR 24 \#A2958; MR 41 \#4467.

6. H. E. Schlais, Non-aposyndesis and non-hereditary decomposability, Pacific J. Math. 45 (1973), 643-652.

7. E. S. Thomas, Jr., Monotone decompositions of irreducible continua, Rozprawy Mat. 50 (1966), 1-74. MR 33 \#4907.

Department of Mathematics, California State University, Chico, California 95926 